\title{
Stellar rotation period inference with Gaussian processes
}

\author{
Ruth Angus $^{1}$, Susanne Aigrain ${ }^{1}$ and Daniel Foreman-Mackey ${ }^{2}$ \\ ${ }^{1}$ Subdepartment of Astrophysics, University of Oxford, Oxford, UK \\ email: ruth.angus@astro.ox.ac.uk, suzanne.aigrain@astro.ox.ac.uk \\ ${ }^{2}$ Sagan Fellow, Department of Astronomy, University of Washington, Seattle \\ email: danfm@uw.edu
}

The light curves of spotted, rotating stars are often non-sinusoidal and Quasi-Periodic (QP) and a strictly periodic sinusoid is therefore not a representative generative model. Ideally, a physical model of the stellar surface would be conditioned on the data, however the parameters of such models can be highly degenerate. Instead, we use an appropriate effective model: a Gaussian Process (GP) with a QP covariance kernel function,

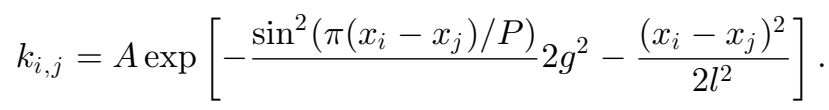

By modelling the covariance matrix of the light curve with a QP GP, we remain agnostic about model choice, whilst sampling directly from the posterior probability distribution function of the periodic parameter and marginalising over the other kernel hyperparameters.

We simulated 300 light curves with a range of rotation periods and spot lifetimes and attempted to recover the rotation periods using three methods: our GP method, a sine-fitting periodogram method and an AutoCorrelation Function (ACF) method (McQuillan et al. 2014). Results are shown in Figure 1. The posterior probability distribution of the rotation period parameter was sampled using the affine invariant ensemble MCMC sampler, emcee (Foreman-Mackey et al., 2013) and the GP operations were performed using the george python package (Foreman-Mackey 2015). This method produces rotation periods that are more precise than the periodogram and both more accurate and precise than the ACF method. Furthermore, the improvement is expected to be even more dramatic when applied to real, noisy Kepler light curves, since the GP method is well suited to modelling rotation signals and correlated noise simultaneously. 


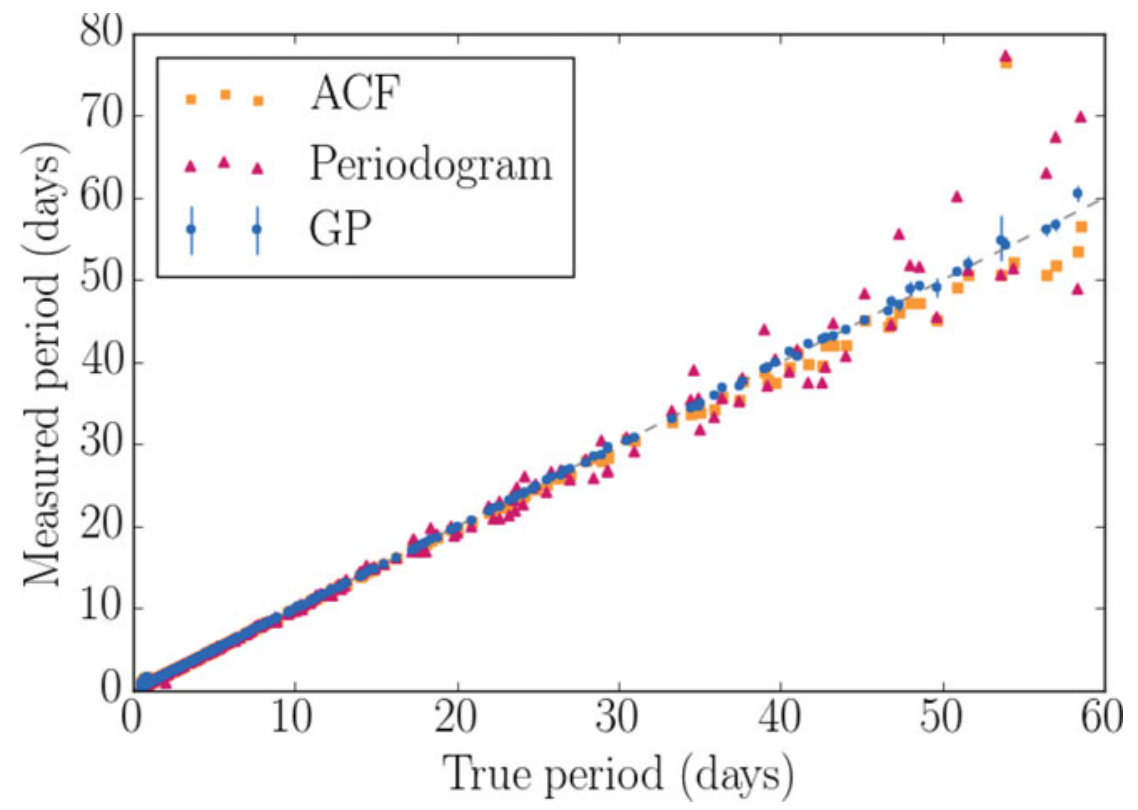

Figure 1. Measured vs true rotation periods for 300 simulations of light curves from spotted, rotating stars. Three different methods were tested: the ACF method, a Lomb-Scargle periodogram (sine-fitting) method and our new GP method. The GP method measures the most precise and accurate rotation periods and is expected to perform even better on real data.

\section{References}

McQuillan, A., Mazeh, T., \& Aigrain, S., 2014, ApJ, 211, 24

Foreman-Mackey, D., Hogg, D. W., Lang, D., \& Goodman, J., 2013, PASP, 125, 306

Foreman-Mackey, D. 2015, https://github.com/dfm/george. 